\title{
THE SPACE DISTRIBUTION OF PLANETARY NEBULAE
}

\author{
J.H. CAHN * \\ (University College London, England)
}

\begin{abstract}
A BSTRACT
A punched-card catalogue of planetary nebulae has been prepared, using data extracted from all existing catalogues. A computer program calculates distances and radii using the method of Shklovsky, in which all nebulae are assumed to have the same ionized mass, and allowance for interstellar extinction is made assuming a continuous galactic-dust distribution. The assumption made in Shklovsky's method, that the nebulae are optically thin, is considered to be satisfied if the calculated radii lie within a certain well-defined interval. The reddening constants obtained are in satisfactory statistical agreement with constants determined by other methods. The local density of planetary nebulae is in agreement with estimates of local white-dwarf densities.
\end{abstract}

A study of the current data on planetary nebulae (Vorontsov-Velyaminov, 1962; O’Dell, 1962; Perek, 1963; Vorontsov-Velyaminov et al., 1964a, b, 1965; Abell, 1966; Henize, 1967; Westerlund and Henize, 1967) has permitted the statistical determination of the distances of 537 planetaries where both angular size and optical flux were available. Preliminary determinations of local density and parameters describing the galactic space distribution are generally in accord with previous determinations.

The method of Shklovsky (Shklovsky, 1956) as modified by Seaton (Seaton, 1966) is used. Briefly, the luminosity $\Lambda$ or the surface brightness $\Sigma$ is expressed in terms of the recombination radiation for an idealized spherical geometry of radius $R$ in the following way:

$$
\Lambda=4 \pi R^{2} \Sigma=\frac{4 \pi R^{3}}{3} \varepsilon \alpha(\lambda) N_{\mathrm{e}}^{2} \frac{h c}{\lambda} .
$$

The electron density $N_{\mathrm{e}}$ is assumed constant over the fraction $\varepsilon$ of the volume and zero elsewhere. $\alpha(\lambda)$ is an effective recombination coefficient for line radiation of wavelength $\lambda$. In the Shklovsky method the mass $M$ of the ionized nebula is assumed constant and replaces the electron density through the relation

$$
M=\frac{4 \pi R^{3}}{3} \varepsilon N_{\mathrm{e}} m_{\text {proton }} \text {. }
$$

Replacing the brightness $\Sigma$ by the flux $F$ at the telescope, corrected for atmospheric extinction, by

$$
R^{2} \Sigma=r^{2} F \times 10^{c_{\lambda}(r)}
$$

* Permanently at University of Illinois, Urbana, Ill., U.S.A. 
where $c_{\lambda}(r)$ is the interstellar extinction and $r$ is the nebular distance, one obtains the implicit equation for $r$,

$$
r^{5}=\frac{3}{\varepsilon}\left(\frac{M}{4 \pi m_{\text {proton }}}\right)^{2} \frac{h c}{\lambda \theta^{3} \times 10^{c_{\lambda}(r)} F},
$$

where $\theta$ is the angular radius. The interstellar extinction, $c_{\lambda}(r)$, is determined by integration of the extinction per unit distance (Perek, 1963), $\alpha_{\lambda} /(1+m)^{2}$, along the path to the planetary

$$
c_{\lambda}(r)=\int_{0}^{r} \frac{\alpha_{\lambda} \mathrm{d} r}{\left(1+m^{2}\right)^{2}}
$$

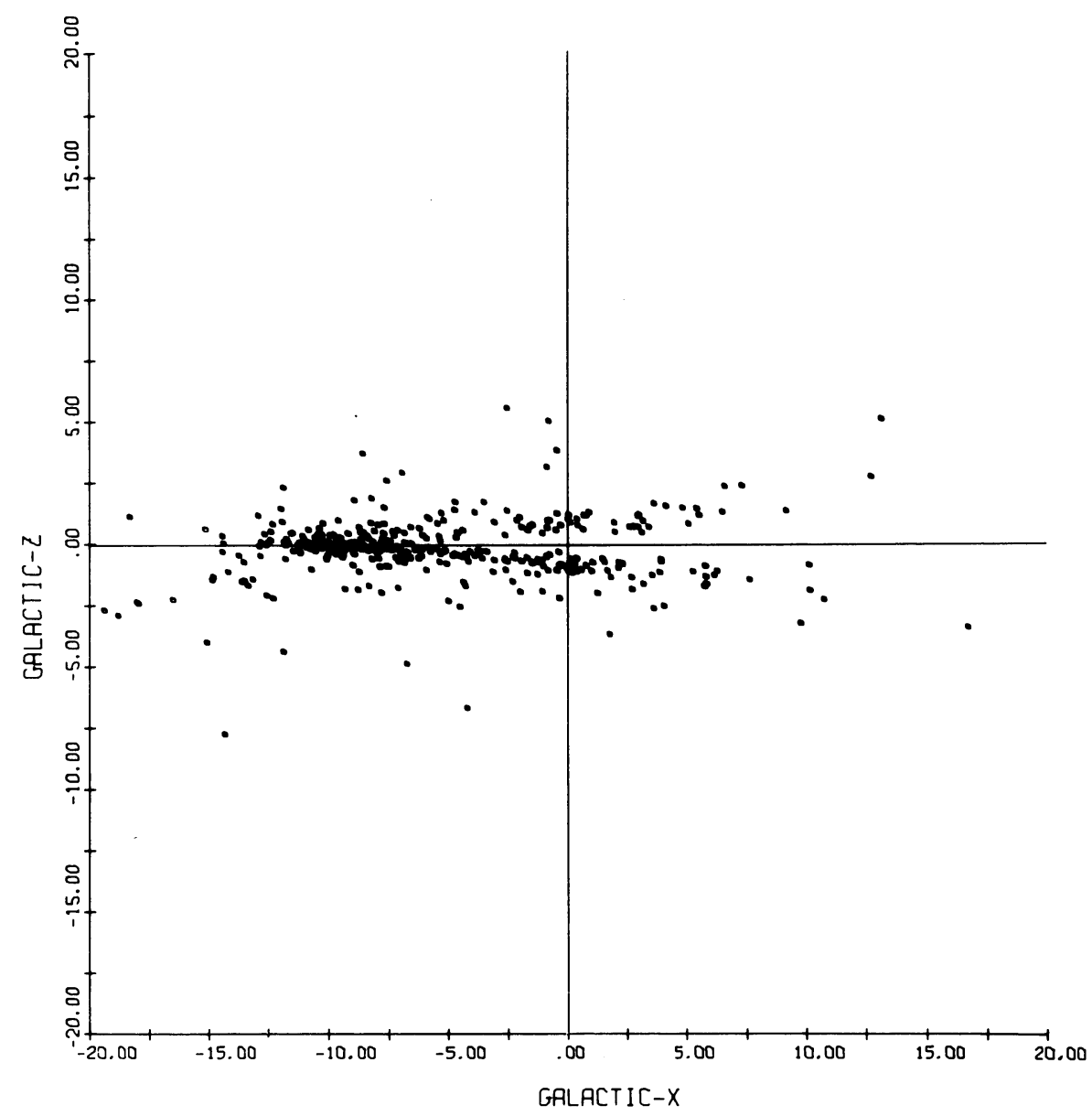

FIG. 1. Projection on $x$-z axis of calculated positions of optically thin planetary nebulae. S represents Sun. The units are in kiloparsecs. 
where $m^{2}$ is the quantity $\left(\rho / a_{\mathrm{d}}\right)^{2}+\left(z / c_{\mathrm{d}}\right)^{2}$ determined from the galactic polar coordinates $\rho, z$ and the scale factors $a_{\mathrm{d}}$ and $c_{\mathrm{d}}$ of the galactic dust distribution.

In order to gain an impression of the adopted distance scale which is based on Seaton's model, $M=0.38 m_{\odot}$ and $\varepsilon=0.63$ (Seaton, 1966), Figures 1 and 2 show the calculated galactic distribution as projected on the $x-z$ and the galactic planes. When the distance scale was varied ( $k=1$ represents the scale of the present calculation) both $k=1.5$ and $k=0.5$ had to be rejected. The case $k=1.5$ was untenable because far too many planetaries were placed beyond the galactic centre, while the case $k=0.5$ gave a heliocentric distribution. The scale $k=0 \cdot 75$, which corresponds approximately to that of O'Dell (O'Dell, 1962), is hardly distinguishable from the scale $k=1$. The effect of the interstellar dust concentration in the galactic plane is shown in Figure 3 in that only planetaries relatively close to the Sun can be observed.

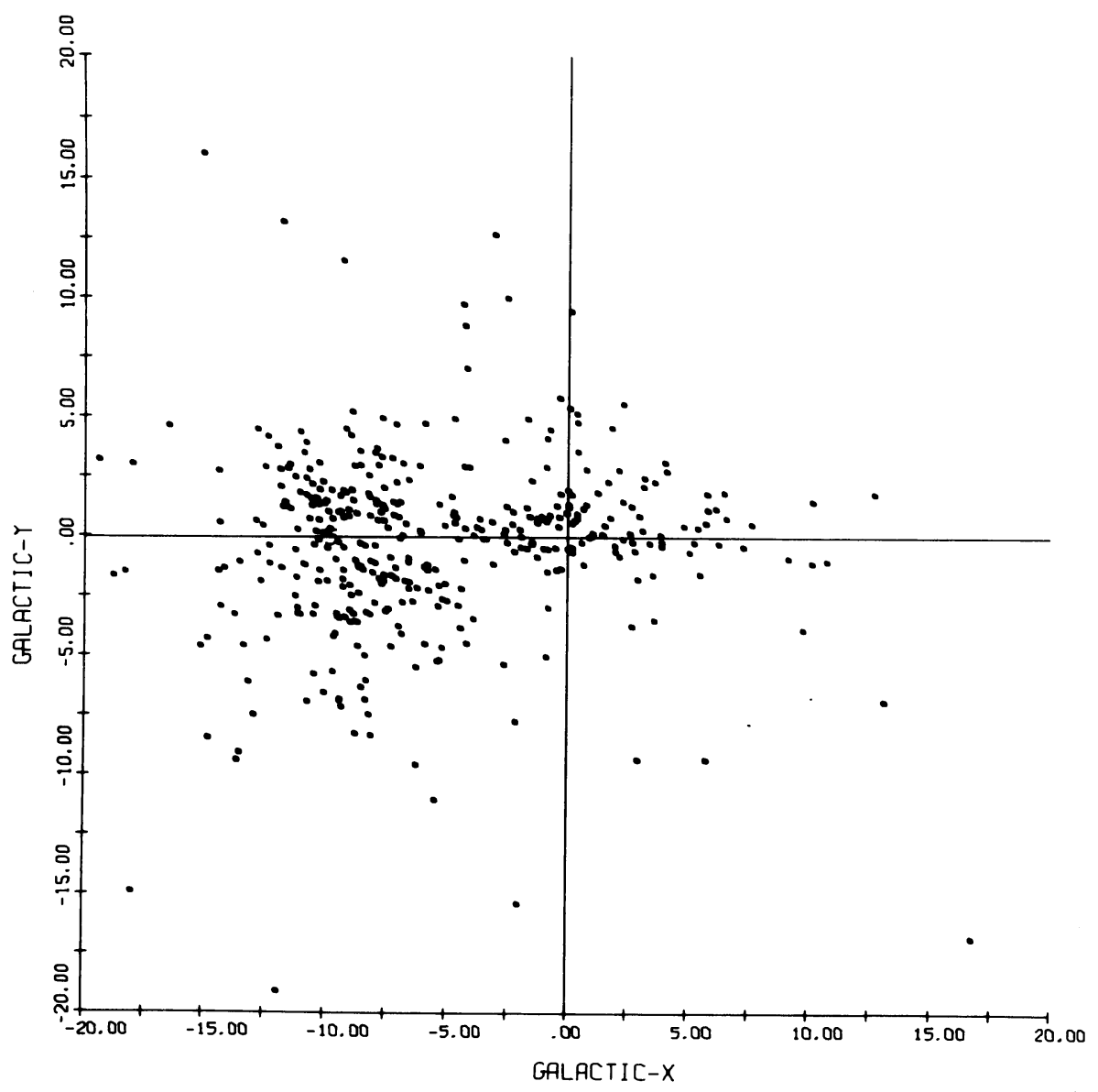

FIG. 2. Projection of optically thin nebulae on galactic plane. 


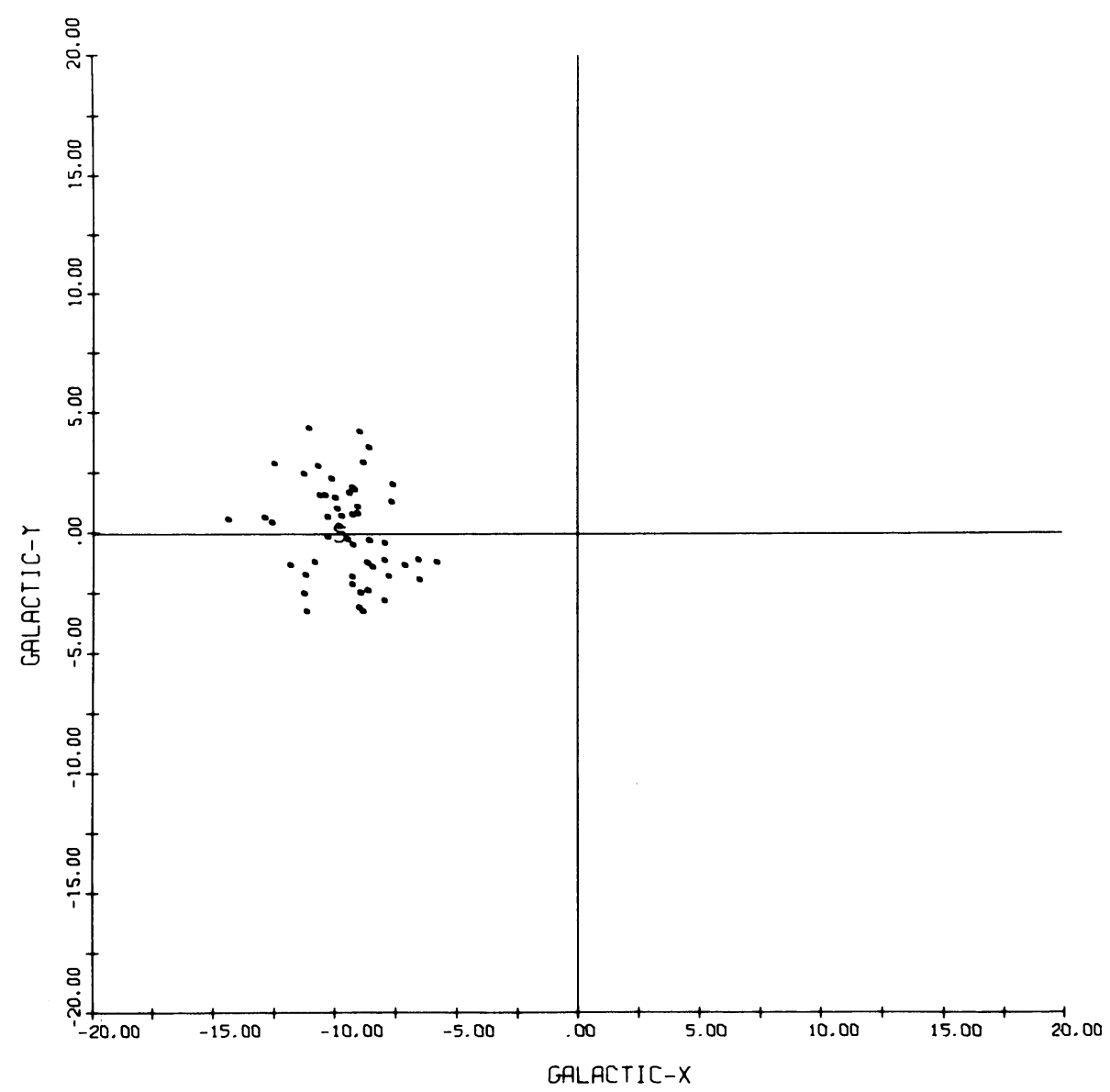

FIG. 3. Projection on galactic plane of those optically thin nebulae within 100 parsec of the galactic plane.

In comparing the extinctions calculated by the method of this paper with measured extinctions, we find, as shown in Figure 4, agreement to about $75 \%$ when comparing with radio and photoelectric data (O'Dell, 1962; Terzian, 1966; Thompson et al., 1967). A rough check on the distance scale is provided by comparing calculated and observed extinctions for scale reductions of factors of 2. Such large reductions are clearly unacceptable when compared with observation. However, calculated extinctions are insensitive to increases of scale factor, since the elongation of the path length in general puts the planetary outside the influence of the galactic dust distribution. The extinction was found to be consistent with the galactic dust distribution adopted by Perek (Perek, 1963).

If the planetaries are undergoing a uniform expansion, there should be, in the 


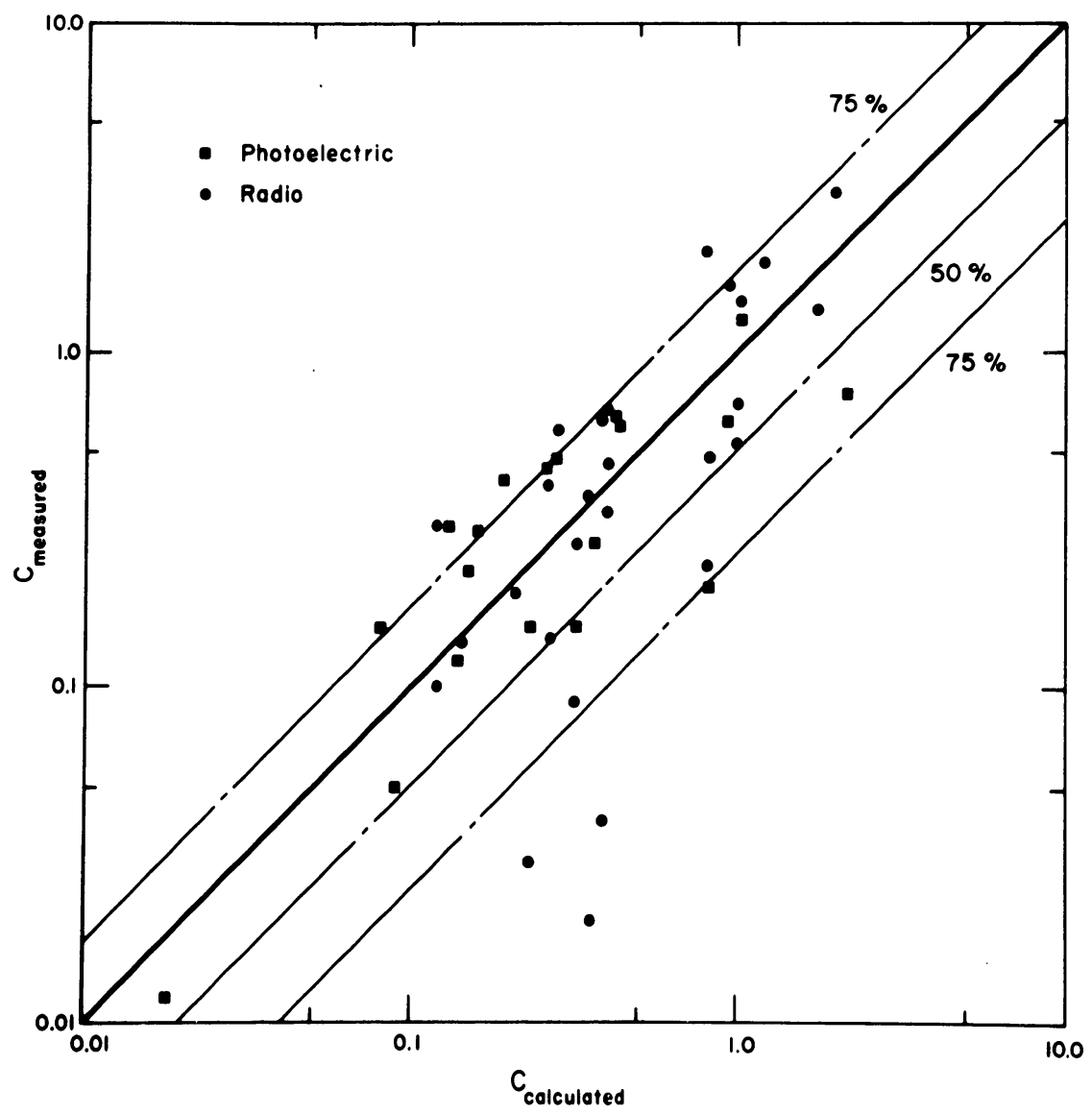

FIG. 4. Comparison of measured and calculated extinctions.

optically thin regime (Seaton, 1966), a constant number per unit radius interval. This is statistically borne out in Figure 5 for those radii $0.12<R<0.60$ parsec as demonstrated by Seaton (Seaton, 1966). Figure 5 shows as well the expected fall-off for larger radii, and the lack of large radii planetaries, due to low surface brightness, beyond $2 \mathrm{kpc}$. By an extrapolation procedure, it is found that the local density of optically thin planetaries in a sphere of zero radius centered at the Sun, $\rho_{L}$, is $12 \mathrm{kpc}^{-3}$. The local number per unit radius interval, $N(R)$, is obtained by dividing the local density, $\rho_{L}$, by 0.48 parsec, the radius range over which the planetaries are optically thin,

$$
\begin{aligned}
N(R) & =\rho_{L} / 4.8 \times 10^{-4} \\
& =2.5 \times 10^{4} \mathrm{kpc}^{-4} .
\end{aligned}
$$

$N(R)$ is the apparent number density per unit radius interval and is equal to the physical density if the Shklovsky method is valid. 

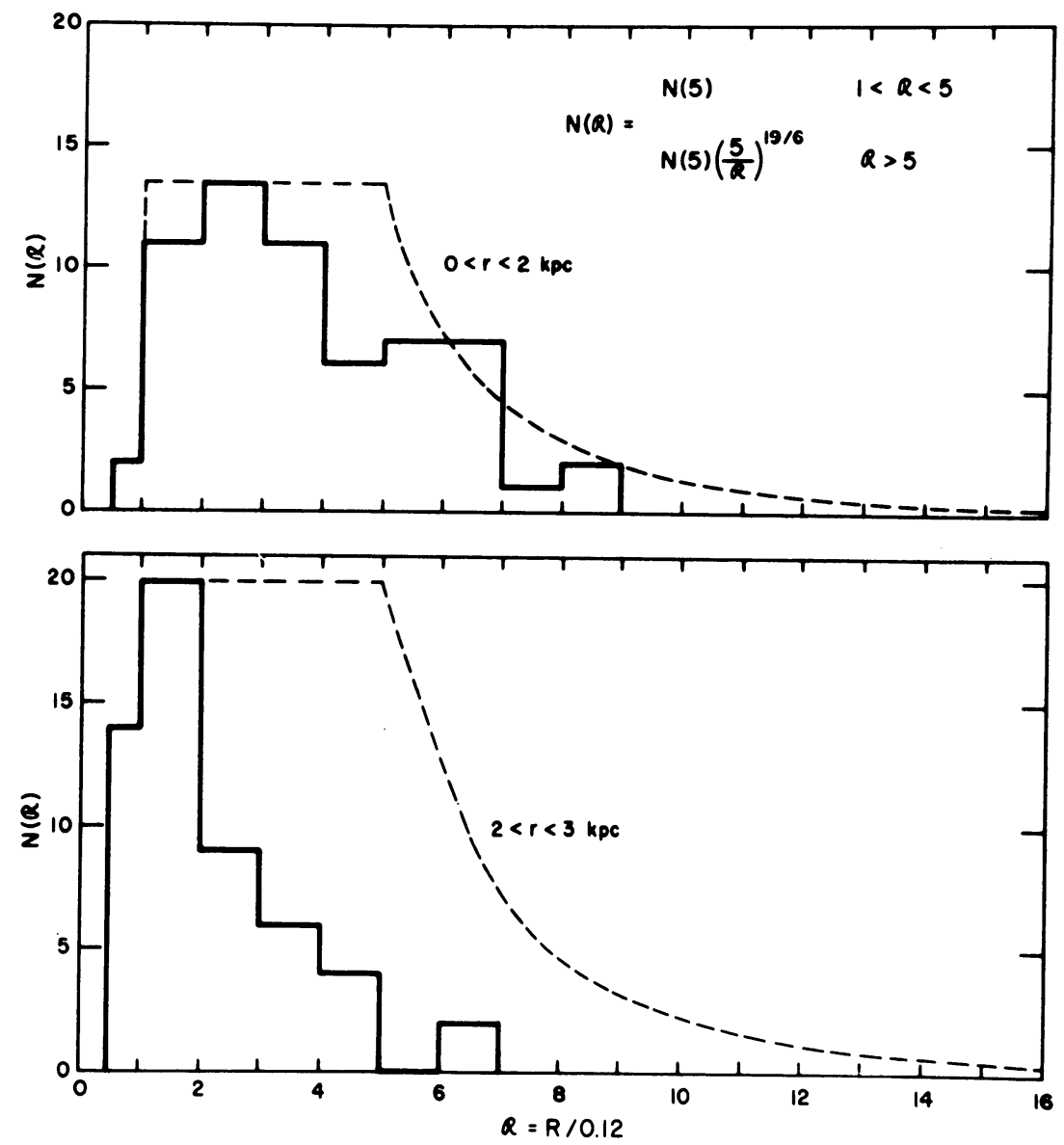

FIG. 5. Statistical distribution of number per unit volume per unit radius, $N(R)$, as a function of reduced radius. The expected fall-off beyond $R=5$ (Seaton, 1966) is shown by the dashed line.

From the number $N(R)$ we compute the local production rate of planetaries, $\chi_{\mathrm{PN}}$, from the expansion velocity $V=20 \mathrm{~km} \mathrm{sec}^{-1}=2 \times 10^{-8} \mathrm{kpc}^{-1} \mathrm{rear}^{-1}$ to be

$$
\chi_{\mathrm{PN}}=V N(R)=5 \times 10^{-4} \mathrm{kpc}^{-3} \mathrm{year}^{-1} .
$$

The local white-dwarf birth rate of $2 \times 10^{-3} \mathrm{kpc}^{-3} \mathrm{year}^{-1}$ (Weidemann, 1968) is seen to exceed the estimated production rate of planetaries by a factor 4 . If scale $k=0.75$ is adopted, one obtains $N(R)=7 \cdot 1 \times 10^{4} \mathrm{kpc}^{-4}$ so that the corresponding production rate $\chi_{\mathrm{PN}}=1.4 \times 10^{-3} \mathrm{kpc}^{-3}$ year $^{-1}$. Thus, within the uncertainty of our distance scale, the white-dwarf population must have received a major contribution from the planetary nebulae. 


\title{
Acknowledgments
}

The author acknowledges the continuing enthusiasm and advice of Professor M.J.Seaton, who suggested this problem. The hospitality of University College London and the financial support of the U.S. Navy are gratefully acknowledged.

\section{References}

\author{
Abell, G.O. (1966) Astrophys. J., 144, 259. \\ Henize, K.G. (1967) Astrophys. J. Suppl. Ser., 14, 125. \\ O'Dell, C.R. (1962) Astrophys. J., 135, 371. \\ Perek, L. (1963) Bull. astr. Inst. Csl., 14, 201. \\ Seaton, M.J. (1966) Mon. Not. R. astr. Soc., 132, 113. \\ Shklovsky, I.S. (1956) Astr. Zu., 33, 222, 315. \\ Terzian, Y. (1966) Astrophys. J. 144, 657.
}

Thompson, A. R., Colvin, R.S., Stanley, G. J. (1967) Astrophys. J., 148, 429.

Vorontsov-Velyaminov, B. A. (1962) Soobšč. gos. astr. Inst. P. K. Šternberga, 118, 3.

Vorontsov-Velyaminov, B. A., Kostjakova, E. B., Dokuchaeva, O.D., Arhipova, V.P. (1964a) Astr. Cirk. SSSR, 305.

Vorontsov-Velyaminov, B. A., Kostjakova,E. B., Dokuchaeva, O. D., Arhipova, V.P.(1964b) Astr. $Z u .$, 41, 255, 464.

Vorontsov-Velyaminov, B. A., Kostjakova, E. B., Dokuchaeva, O.D., Arhipova, V.P.(1965) Astr. $Z u ., 42,730$.

Weidemann, V. (1968) in the present volume, p. 423.

Westerlund, B. E., Henize, K.G. (1967) Astrophys. J. Suppl. Ser., 14, 154.

\section{DISCUSSION}

Feast: Cahn has discussed the effects of systematic changes in the distance scale. However, one also has to consider the systematic errors in the distances introduced by the random errors in individual distance estimates. This is a statistical problem which may result in considerable correction being required, especially for the mean distance of the most distant objects.

Cahn: I estimate the errors to be at most a factor of 2 . The distribution function was determined by fitting only near the Sun where the discovery is reasonably complete. 